\title{
SOME REMARKS ON CONTINUOUS TRANSFORMATIONS
}

\author{
V. L. KLEE AND W. R. UTZ
}

Suppose $f$ is a single-valued transformation of the metric space $M$ onto the metric space $f M$. Many of the theorems of topology assert that if $f$ is continuous, then it must have various other properties also. We deal here with the problem of determining under what circumstances certain of these properties are actually equivalent to continuity. The properties considered are:

(1) $f X$ is compact for every compact $X \subset M$;

(2) $f Y$ is connected for every connected $Y \subset M$;

(3) $f^{-1} q$ is closed for every point $q \in f M$;

(4) $f^{-1}\{z: z \in f M$ and $\rho(z, q)=\epsilon\}$ is closed for each $\epsilon>0$ and $q \in f M$.

By an " $i$-map" on $M$ we will mean a transformation $f$ satisfying the condition $(i)$, where $i=1,2,3$, or 4 .

A basic lemma is

(L) Suppose $f$ is a 1-map on $M$, discontinuous at the point $p \in M$. Then there are a point $q \in f M$ and a sequence $p_{\alpha}$ of points of $M$ such that $p_{\alpha} \rightarrow p, q \neq f p$, and $f p_{i}=q$ for each $i$.

Proof. Clearly there are an open set $V \ni f p$ and a sequence $x_{\alpha}$ of distinct points of $M$ such that $x_{\alpha} \rightarrow p$ and $f x_{i}=q_{i} \notin V$. If the desired conclusion fails to hold, then each $q_{i}$ is the image of only finitely many $x_{i}$ 's, and there is an infinite subsequence $y_{\alpha}$ of $x_{\alpha}$ such that $S=\left\{y_{1}, y_{2}, \ldots\right\}$ maps biuniquely under $f$. But $S \cup\{p\}$ is compact, so $f S \cup\{f p\}$ is compact, and hence, since $f S$ misses $V, f S$ is compact. Thus for some $j, f y_{j}$ is an accumulation point of $f S$. But $\left(S-\left\{y_{j}\right\}\right)$ $\cup\{p\}$ is compact and hence (as above) $f S-\left\{f y_{j}\right\}$ is compact, a contradiction completing the proof.

Since $f^{-1} q$ in (L) is clearly not closed, we have

(A) Every 1, 3-map on $M$ is continuous.

A corollary is the fact that every biunique 1-map is continuous.

Our next result is

(B) If $M$ is locally connected at $p$, then every $1,2-$ map on $M$ is continuous at $p$. If $M$ is not locally connected at $p$, then $M$ admits a realvalued 1, 2-map which is discontinuous at $p$.

Proof. To prove the first assertion, suppose $M$ is locally connected at $p$ but admits a 1, 2-map $f$ (onto some metric space) which is discontinuous at $p$. Let $p_{\alpha}$ and $q$ be as in (L). By local connectedness of $M$ at $p$, there are a subsequence $x_{\alpha}$ of $p_{\alpha}$ and a sequence $C_{\alpha}$ of con1953.

Presented to the Society, November 28, 1953; received by the editors July 14, 
nected subsets of $M$ such that always $\left\{p, x_{i}\right\} \subset C_{i} \subset N_{1 / i} p$. But $\{p, q\} \subset f C_{i}$, which must be connected so $f C_{i}$ contains a point $z_{i}$ such that $0<\rho\left(q, z_{i}\right)<1 / i$. For each $i$, let $u_{i} \in C_{i} \cap f^{-1} z_{i}$. Then $\left\{p, u_{1}, u_{2}, \cdots\right\}$ is compact but its image is not, a contradiction completing the proof of the first assertion.

Now suppose $M$ is not locally connected at $p$. Then there is a $\delta \in] 0,1$ [ such that $p$ is not interior to the $p$-component $Q$ of $N_{2 \delta} p$. Let $f x=0$ for $x \in M-N_{2 \delta} p, f x=2 \delta-\rho(x, p)$ for $x \in N_{2 \delta} p-N_{\delta} p, f x$ $=\rho(x, p)$ for $x \in N_{\delta} p \cap Q$, and $f x=\delta$ for $x \in N_{\delta} p-Q$. Let $A=Q$ $\cup\left(M-N_{\delta} p\right)$. Then $f \mid A$ and $f \mid M-N_{\delta} p \cap Q$ are continuous, but $f \mid M$ is discontinuous at $p$. We must show that $f$ is a 1,2-map. Consider an arbitrary compact $X \subset M$. Then $f(X \cap A)$ is compact, for $A$ is closed and $f \mid A$ continuous. And $f(X-A) \in\{\delta\}$, so $f X$ must be compact and $f$ is a 1-map. Now consider an arbitrary connected $Y \subset M$. Since $f \mid A$ and $f \mid M-N_{\delta} p \cap Q$ are continuous, $f Y$ is surely connected if $Y$ is contained in either of these sets. In the remaining case, $Y$ intersects both $N_{\delta} p \cap Q$ and $N_{\delta} p-Q$ and, being connected, is not contained in $N_{2 \delta} p$. Hence $\{\rho(y, p): y \in Y\} \supset[\delta, 2 \delta]$, whence $f Y=[0, \delta]$. Thus $f$ is a 2-map and the proof of (B) is complete.

Before proceeding, we remark that every 4-map is a 3-map and that every real-valued 3-map is a 4-map.

(C) For the point $p \in M$, the following assertions are equivalent: (i) $M$ is locally connected at $p$; (ii) every 2, 4-map on $M$ is continuous at $p$; (iii) every real-valued 2, 3-map on $M$ is continuous at $p$.

Proof. Clearly (ii) implies (iii). To see that (i) implies (ii), suppose $M$ is locally connected at $p$ and consider arbitrary $\eta>0$ and 2, 4map $f$ on $M$. Let $B_{\eta}=\{z: z \in f M$ and $\rho(z, f p)=\eta\}$. Then $f^{-1} B_{\eta}$ is closed and misses $p$, so by local connectedness at $p$ there is a connected set $U \subset M-f^{-1} B_{\eta}$ such that $p$ is interior to $U$. But $f U$ is connected, contains $f p$, and misses $B_{\eta}$, whence $f U \subset N_{\eta} f p$, showing that $f$ is continuous at $p$. Thus (i) implies (ii). To see that (iii) implies (i), suppose $M$ is not locally connected at $p$ and let $\delta, Q$ and $f \mid A$ be as in the proof of (B). For $x \in M-A=N_{\delta} p-Q$, let $f x=\delta-[\delta-\rho(x, p)] \rho(x, Q)$. It can be verified that $f$ is a 2,3-map which is discontinuous at $p$. Thus (iii) implies (i) and the proof of (C) is complete.

For the case in which $M$ is the real line, that (i) implies (iii) was proved by $\mathrm{C}$. $\mathrm{H}$. Rowe [Note on a pair of properties which characterize continuous functions, Bull. Amer. Math. Soc. vol. 32 (1926) pp. 285$287]$. He also gave an example of a noncontinuous real-valued 2, 3-map.

Now let $\phi$ be an arbitrary real-valued function on $[0, \infty$ [ such that the graph $G_{\phi}=\{(r, \phi r): r \in[0, \infty[\}$ is connected but $\phi$ is dis- 
continuous at 0 . (For example, $\phi x=\sin (1 / x)$ for $x>0$ and $\phi 0=0$.)

(D) The point $p \in M$ is an accumulation point of $M$ if and only if $M$ admits a 2, 3-map into $G_{\phi}$ which is discontinuous at $p$.

Proof. The "if" part being obvious, we shall consider the "only if" part. If $p$ is an accumulation point of $M$, there is a sequence $p_{\alpha} \rightarrow p$ such that $\rho\left(p_{1}, p\right)>\rho\left(p_{2}, p\right)>\cdots$. Since $\phi$ is discontinuous at 0 , there is a sequence of numbers $r_{1}>r_{2} \cdots$ such that $r_{\alpha} \rightarrow 0$ but $\phi r_{\alpha}$ does not converge to $\phi 0$. There is a homeomorphism $h$ of $[0, \infty$ [ onto itself such that $h \rho\left(p_{i}, p\right)=r_{\boldsymbol{i}}$ for each $i$. Now for each $x \in M$ let $f x$ $=(h \rho(x, p), \phi h \rho(x, p))$, the image $f M$ being assigned the usual plane topology. It can be verified that $f$ has the desired properties.

Related results have been obtained by Ky Fan and Raimond A. Struble, Continuity in terms of connectedness, to appear in Neder. Akad. Wetensch.

UNIVERSITY OF VIRGINIA AND

UNIVERSITY OF MISSOURI 